\title{
A Novel PRRT2 Variant in Chinese Patients Suffering from Paroxysmal Kinesigenic Dyskinesia with Infantile Convulsion
}

\author{
Salem Baldi $\mathbb{D},{ }^{1}$ Jin-Ling Zhu $\mathbb{D},{ }^{1}$ Qing-Yun Hu $\mathbb{D}^{2},{ }^{2}$ Ju-Li Wang $\mathbb{D},{ }^{3}$ Jin-Bo Zhang $\mathbb{D},{ }^{1}$ \\ and Shu-Hong Zhang ${ }^{1}$ \\ ${ }^{1}$ Department of Biology, School of Basic Medicine, Jiamusi University, Jiamusi City, Heilongjiang Province, 154007, China \\ ${ }^{2}$ Department of Anatomy, School of Basic Medicine, Jiamusi University, Jiamusi City, Heilongjiang Province, 154007, China \\ ${ }^{3}$ Department of Paediatrics, Central Hospital of Jiamusi, Jiamusi City Heilongjiang Province, 154002, China
}

Correspondence should be addressed to Jin-Ling Zhu; 13845414150@139.com

Received 24 December 2019; Accepted 20 April 2020; Published 18 May 2020

Academic Editor: Enzo Emanuele

Copyright (c) 2020 Salem Baldi et al. This is an open access article distributed under the Creative Commons Attribution License, which permits unrestricted use, distribution, and reproduction in any medium, provided the original work is properly cited.

\begin{abstract}
PRRT2 mutations are the major causative agent of paroxysmal kinesigenic dyskinesia with infantile convulsion (PKD/IC). The study is aimed at screening PRRT2 gene mutations in patients who suffered from PKD/IC in Chinese population. Thirteen Chinese patients with PKD/IC were screened randomly for coding exons of the PRRT2 gene mutation along with 50 ethnically coordinated control people. Nine ( 2 unaffected) and 4 of the patients showed familial PKD/IC and apparently sporadic cases, respectively. We identified 5 different PRRT2 mutations in 10 individuals, including 8 familial and 2 apparently sporadic cases. However, no mutations were found in the 50 ethnically matched controls. Unknown (novel) NM_145239.2:c.686G>A and previously reported NM_145239.2:c.743G $>$ C variants were identified in two familial and sporadic patients. All affected members of family A showed mutation NM_145239.2:c.650_670delinsCAATGGTGCCACCACTGGGTTA. The previously identified NM_145239.2:c.412 C>G and NM_145239.2:c.709G >A variants are seen in two individuals assessed in family B. Other than the previously identified variants, some of the patients with PRRT2-PKD/IC showed a new PRRT2 substitution variant. Thus, the spectrum of PRRT2 variants is expanded. The possible role and probability of PRRT2 variants involved in PKD/IC are highlighted.
\end{abstract}

\section{Introduction}

The paroxysmal dyskinesia comprises movement disorders characterized by decrease voluntary movements and the presence of involuntary movements. Three main types are present and categorized according to duration and recurrence of the attacks, namely, paroxysmal kinesigenic dyskinesia (PKD), paroxysmal nonkinesigenic dyskinesia, and paroxysmal exercise-induced dyskinesia, which are exertion-induced. Initially, PKD was the only known disorder characterized by unilateral or bilateral involuntary movement induced by sudden movement from rest, such as starting to walk or ascending from the sitting position. The attacks last for less than $1 \mathrm{~min}$ in duration with no pain or loss of consciousness. PKD, convulsions, familial infantile, with paroxysmal choreoathetosis (PKD/IC) and seizures, and benign familial infantile 2 (BFIS2) were recently linked to a similar district region 16p12-q12 and considered to be allelic diseases [1]. The PRRT2 gene provides instruction for the creation of the PRRT2 protein. The synaptic function is regulated by this protein by interacting with the synptosomal-associated protein (SNAP25) and different molecules of the $\alpha$-amino-3-hydroxy-5-methyl-4isoxzazolepropionic acid (AMPA) complex receptors. The structural changes in PRRT2 gene sequence, such as base pair substitution, insertion, and deletion, can generate different types of mutations, including missense and nonsense mutations. The PRRT2 gene situated on the chromosome 16p11.2 with 4 exons encodes a membrane protein and reportedly causes several nervous system diseases [3]. PRRT2 protein occurrence is important in developing the sensory system, which includes the base of the brain and ganglia, throughout the embryonic developmental stages [4]. The PRRT2 has been depicted in various independent groups of Asian, African, 


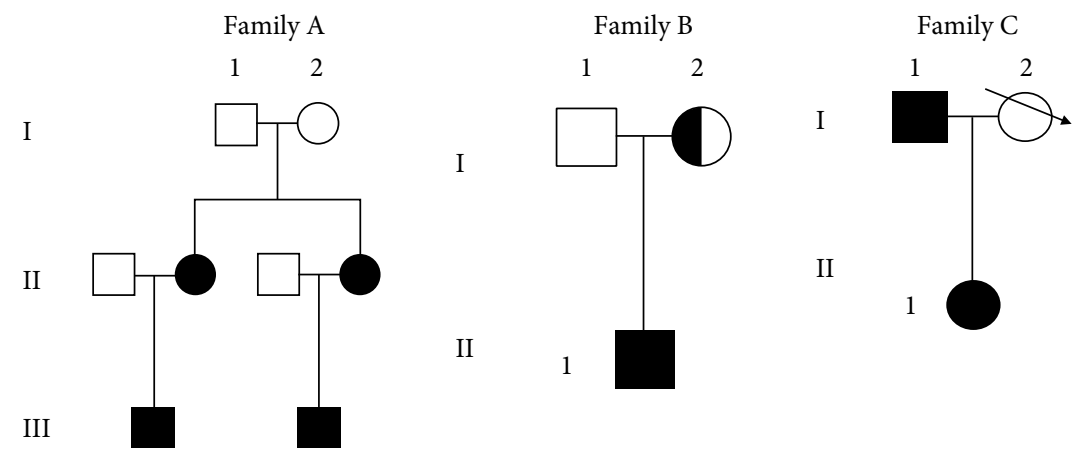

Figure 1: PKD/IC family pedigrees. The NM_1452392.2:c.650_670delinsCAATGGTGCCACCACTGGGTTA p.(Arg217Profs*8) was found in family A. The NM_1452392.2:c.709G>A p.(Gly237Arg) was found in family B. The NM_1452392.2:c.686G>A p.(Arg229 Lys) was found in family $\mathrm{C}$.

and Caucasian families affected with $\mathrm{PKD}, \mathrm{PKD} / \mathrm{IC}$, and BFIS2 [5-12]. Moreover, other epileptic phenotypes, including febrile seizure-related epilepsy [13-15] and hemiplegic migraine [16-19], have been implicated. We investigated whether the PRRT2 mutations are involved in PKD/IC and discover possible novel genetic mutations. Understanding PRRT2 mutation may reveal new treatment modalities, which may allow physicians to adopt new strategies for effective and timed treatment.

\section{Material and Methods}

2.1. Patients. Thirteen individuals that comprise 9 familial cases (Figure 1) and 4 sporadic cases (Table 1) and 50 healthy controls were recruited from January 2016 to January 2017 in the ward of the Epilepsy Department of the Jiamusi Central Hospital. The controls were used to establish the baseline to compare the result of experiment's subjects and return valid results. All enrolled participants were personally interviewed and evaluated by at least two epileptologist. All the selected patients were new cases and met the diagnostic criteria of PKD set by Brun et al. These criteria include recognizing a kinesgenic trigger, having no pain or loss of consciousness during attacks that last less than 1 minute, and having favorable response to antiepileptic medicated treatment. A case was excluded if the patient did not take the test for PRRT2 gene and refused to provide informed consent for the study. All enrolled patients provided a written consent form in order to utilize their DNA. The study was approved by Medical Ethics Committee of Jiamusi University.

2.2. Mutation Analysis. The peripheral blood samples were collected from each subject, and a BioTeke DNA blood kit was used according to the instructions of the manufacture (BioTeke Corporation, China) to extract genomic DNA. The National Centre for Biotechnology Information (NCBI) software was used to design gene-specific primers. Firstly, DNA was amplified using a Biosystem polymerase chain reaction (PCR) over a number of cycles. PCR experiments were conducted with the following typical cycling conditions: initial denaturation at $95^{\circ} \mathrm{C}$ for $5 \mathrm{~min}$, then 25 cycles of denaturation at $94^{\circ} \mathrm{C}$ for $30 \mathrm{~s}$, annealing at $56^{\circ} \mathrm{C}$ for $30 \mathrm{~s}$, extension at $72^{\circ} \mathrm{C}$ for $45 \mathrm{~s}$, and final extension at $72^{\circ} \mathrm{C}$ for $5 \mathrm{~min}$. We obtained the amplified PCR products that were separated on agarose gels and eventually sequenced using a DNA automated sequencer (Shenggong Bioengineering Co., Ltd., Shanghai, China) to confirm the presence of mutations. The methods were repeated three times and mutation confirmed by reverse sequence. Statistical analysis was performed using one-way ANOVA at the 5\% level of significance. The obtained DNA sequences were contrasted with the genomic reference sequence of PRRT2 mRNA (NM_145239.2) published in NCBI. Thus, the diseasecausing mutant sequence was determined. By the way, the reverse sequence was applied for the positive samples in order to confirm the presence of mutation. The description of variants and predicted proteins were described following current Human Genome Variation Society (HGVS) recommendations (http://www.HGVS.org/varnomen). The Mutalyzer was used to generate and check the HGVS variants description. (http://www.LOVD.nl/mutalyzer). The welldescribed and reported variants were submitted to the PRRT2 gene variant database (Leiden Open Variation Database (LOVD)) [20-24].

2.3. Statistical Analysis. One-way analysis of variance (ANOVA), using Tukey test and NSTAT software was used to determine whether there are any statistically significant differences between the samples. Positive PKD patient group was compared with the negative group to determine the significance, at 0.05 significance level (Table 2).

The correlation and significance of collected data were evaluated by the IBM SPSS by one-way ANOVA. The significance was tested at 0.05 degrees of freedom, where simple $T$ test was used to check the significance of the data.

\section{Results}

3.1. Clinical Features. The main manifestations of each family in our study are different from each other; the common clinical features of PKD, such as the duration of attacks, loss of consciousness during attacks, and good response to carbamazepine treatment, are shared in the three analyzed families. The significant difference between the group with and without PRRT2 gene mutations at the 0.05 level of significance (Table 2) was presented. 


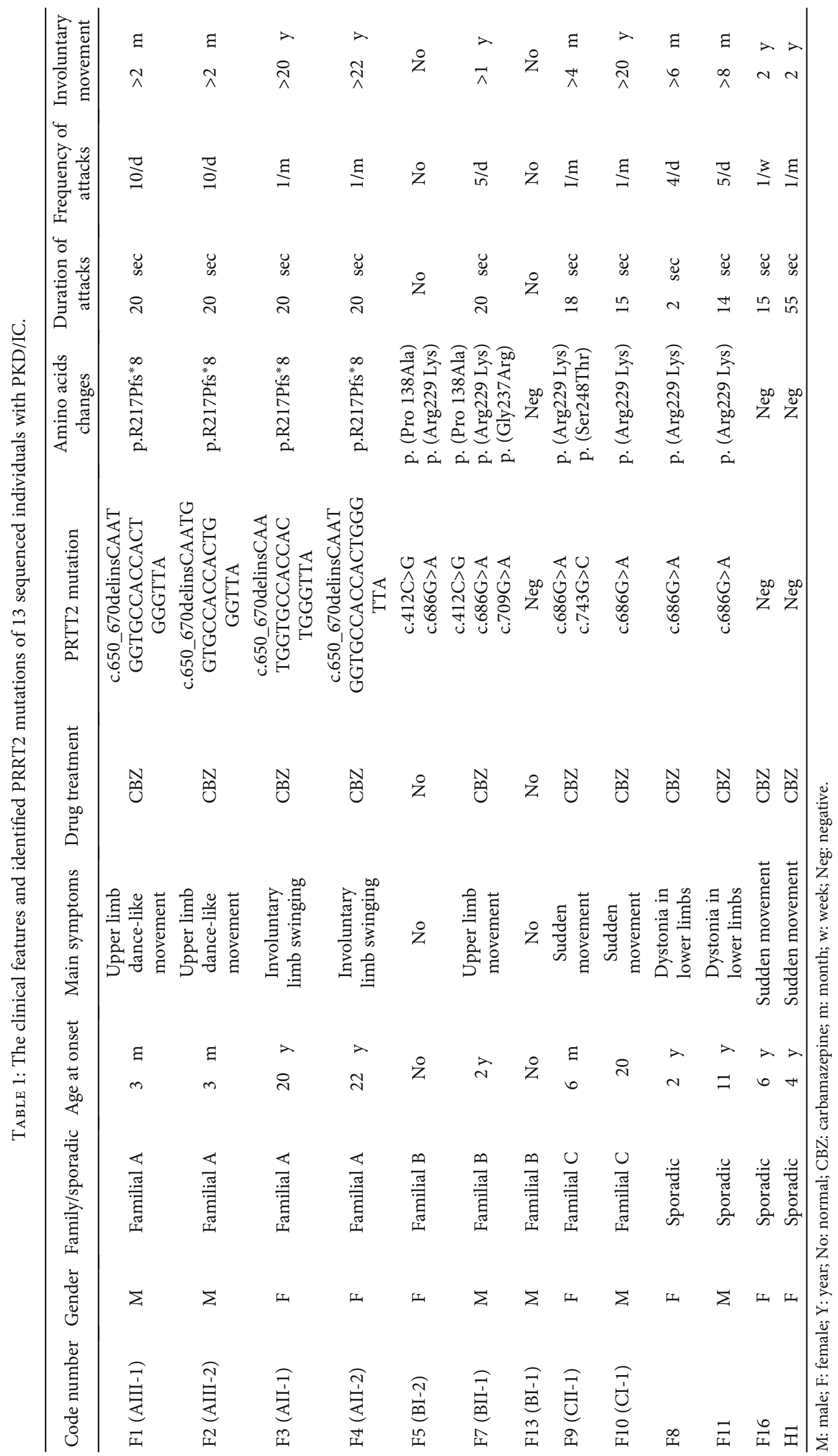


TABLE 2: Statistical analysis and clinical correlations.

\begin{tabular}{lcccccc}
\hline Variable & & Total $(N=13)$ & PRRT2 + VE $(N=10)$ & PRRT2-VE $(N=3)$ & Correlation & $P$ value \\
\hline Age of onset & Mean $(\mathrm{SD})$ & $8 \mathrm{y}(1.318)$ & $8.6 \mathrm{y}(1.924)$ & $5 \mathrm{y}(2.775)$ & -0.3675 & 0.07958 \\
Gender & Median & $6 \mathrm{y}$ & $2 \mathrm{y}$ & $5 \mathrm{y}$ & - & - \\
Male & & & & & & \\
Female & $N(\%)$ & $6(46 \%)$ & $5(50 \%)$ & $1(33.3 \%)$ & -0.4158 & 0.2656 \\
Origin (Chinese) & & $7(53.8 \%)$ & $5(50 \%)$ & $2(66.6 \%)$ & 0.68047 & 0.3504 \\
Family history of PKD & $N(\%)$ & $13(100 \%)$ & $10(77 \%)$ & $3(23 \%)$ & -0.3675 & 0.07958 \\
History of involuntary movement & $N(\%)$ & $7(69 \%)$ & $8(61.5 \%)$ & $1(7.7 \%)$ & -0.395563 & 0.0268 \\
Duration of attack(s) & Mean Median & $15 \mathrm{~s} 20 \mathrm{~s}$ & $12.620 \mathrm{~s}$ & $23.3 \mathrm{~s} 35 \mathrm{~s}$ & 0.427347 & 0.0225 \\
Frequency of attacks & & & & & \\
$1-5$ per day & & $5(38.4 \%)$ & $5(50 \%)$ & $0(0 \%)$ & - & - \\
$1-5$ per week & $N(\%)$ & $1(07 \%)$ & $0(0 \%)$ & $1(33.3 \%)$ & - & - \\
$1-5$ per month & $5(38.4 \%)$ & $4(40 \%)$ & $1(33.3 \%)$ & -0.5222 & 0.12103 \\
\hline
\end{tabular}

Statistical analysis indicates that the significant difference is between positive and negative PKD patients at 0.05 significant level and there is no correlation observed between different variant samples of positive and negative PKD patients.

3.2. Genetic Finding. The idiopathic PKD is a neurodevelopmental disorder firmly affected by genetic factors according to sequence analysis results. Table 1 provides a summary of the clinical features and detected PRRT2 mutations of all familial and sporadic patients suffering from PKD/IC. Eleven patients of $\mathrm{PKD} / \mathrm{IC}, 2$ unaffected relatives, and 50 healthy matched controls were analyzed for PRRT2 mutations. Several previously identified variants and one novel variant were obtained. Of the 3 families with PKD (7 patients, 2 unaffected), 8 individuals (88\%) were identified with PRRT2 mutations. Out of the 4 sporadic cases, 2 (50\%) demonstrated novel PRRT2 mutation. By using the direct sequence of the entire PRRT2 gene, one deletion-insertion mutation and 4 substitution mutations were identified (Figures 2(a)-2(e)) in 10 individuals, including 8 familial (one asymptomatic carrier) and 2 apparently sporadic cases (Table 1). NM_ 1452392.2: c.650_670delinsCAATGGTGCCACCACTGGG TTA p. (Arg217Profs*8) mutation was identified in all patients from family A characterized by involuntary movements and responding appropriately to carbamazepine treatment (Figure 2(a)). The previously known variants of PRRT2 NM_1452392.2: c.412C>G p. (Prol138Ala) (Figure 2(b)) and PRRT2 NM_1452392.2: c.709G>A p. (Gly237Arg) (Figure 2(c)) were also identified in 2 individuals of family B. The previously unknown NM_1452392.2: c.686G $>$ A p. (Arg229Lys) variant (Figure 2(d)) was detected in 6 individuals, of which 2 were in families B and C, and 2 sporadic cases. Polyphen- 2 predicted the case to be benign. The PRRT2 NM_1452392.2: c.743G $>$ C p. (Ser248Thr) (Figure 2(e)) was found in one patient of family $\mathrm{C}$ (Table 1). The severity prediction of NM_1452392.2: p. (Ser248Thr) is most likely harmed by the mutation taster polyphen-2. This finding reveals its pathogenic nature. None of the identified mutations were found in the 50 age-, sex-, and geographically matched healthy controls. Clinicogenetic associations analysis showed that there were no significant difference association of age of onset between patient who carried the mutation compared with those who did not $P=$
0.079 (Table 2). The presence of family history of PKD and history of involuntary movement was not significantly associated with the presence of PRRT2 mutation (Table 2).

\section{Discussion}

PKD is the most widely recognized type of paroxysmal dyskinesia characterized by involuntary convulsion and variability in manifestations [25]. PKD incidence is rare in the population, which accounted to $1 / 150,000$ worldwide. Genomewide linkage analysis and fine-mapping have enabled identification of susceptibility genes, including PRRT2 in PKD/IC appearance [26, 27]. The PRRT2 gene encodes transmembrane protein consisting of $\mathrm{N}$-terminal extracellular and Ccytoplasmic domains [2]. Two recent studies have shown that the PRRT2 is profoundly expressed in neural cell development during the early embryonic stages. Thus, PRRT2 plays a major role in synaptic function. Reduced gene expression or changes in PRRT2 levels are correlated with alteration in neuronal function [28, 29]. Sanger sequencing was used to confirm the occurrence of PRRT2 mutation in 13 patients suffering from $\mathrm{PKD} / \mathrm{IC}$ disorder in the Chinese cohort. The NM_1452392.2: c.650_670delinsCAATGGTGCCACCACT GGGTTA variant introducing a premature stop codon was found in all available members of family A. Thus, supreme cosegregation of PKD incident is shown within the family. This finding is consistent with the hereditary qualities, including the autosomal dominant property of PKD. The common NM_1452392.2: c.649dupC p. (Arg217Profs*8) was previously identified in $\mathrm{PKD} / \mathrm{IC}$ patients $[4,9,30]$. The NM_1452392.2:c.649delC and NM_1452392.2:c.649C>G mutations were previously identified in PKD European population [31].

The p.(Arg217Pfs*8) accounts for $30.8 \%$ of sample population (44.4\%, 4 out of 9 of our familial study population). Our finding and the finding of NM_1452392.2: c.649delC, NM_1452392.2: c.649C>G and NM_1452392.2: c.649dupC at the same position indicate the presence of a mutation 
Normal sequences

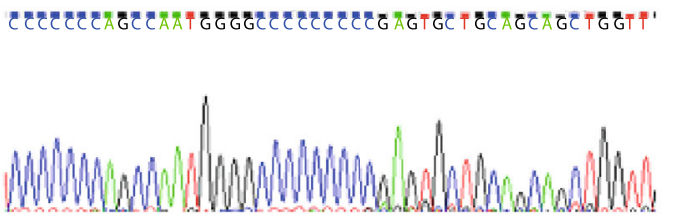

AACCAGCCCCAGAGCCTGCTCCCCAACCAGACCCCCGGCCAGATT

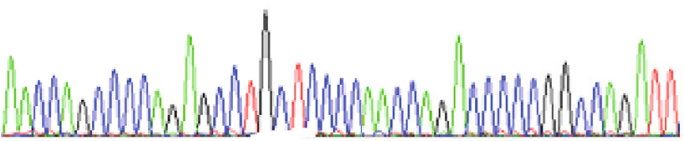

$\bar{A} \bar{A} \bar{A} \bar{G} \bar{G} \bar{G} \bar{C} \bar{A} \bar{C} \bar{A} \bar{A} \bar{C} \bar{A} \bar{G} \bar{T} \bar{G} \bar{G} \bar{G} \bar{C} \bar{A} \bar{T} \bar{C} \bar{C} \bar{A} \bar{G} \bar{G} \bar{A} \bar{A} \bar{T} \bar{C} \bar{T} \bar{C} \bar{C} \bar{C} \bar{C} \bar{G} \bar{A} \bar{G} \bar{G} \bar{T} \bar{T} \bar{G} \bar{G} \bar{C} \bar{C} \bar{T}$.

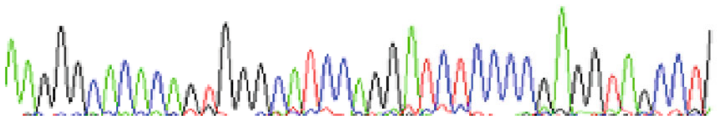

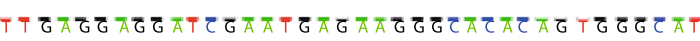

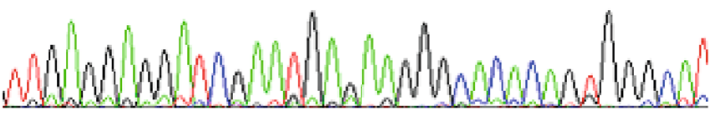

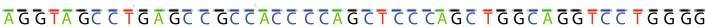

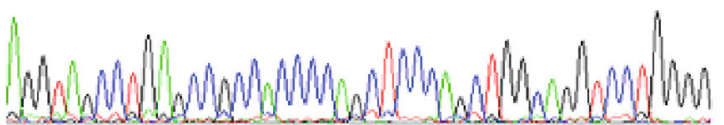

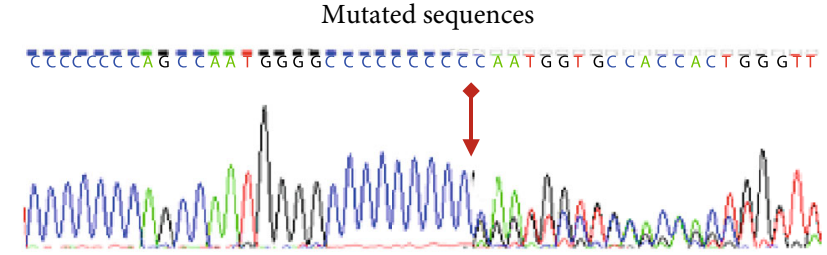

(a)

$\bar{A} \bar{A} \bar{C} \bar{C} \bar{A} \bar{G} \bar{C} \bar{C} \bar{C} \bar{C} \bar{A} \bar{G} \bar{A} \bar{G} \bar{G} \bar{C} \bar{T} \bar{G} \bar{C} \bar{T} \bar{C} \bar{C} \bar{C} \bar{C} \bar{A} \bar{A} \bar{C} \bar{C} \bar{C} \bar{G} \bar{A} \bar{C} \bar{C} \bar{C} \bar{C} \bar{C} \bar{G} \bar{G} \bar{C} \bar{C} \bar{A} \bar{G} \bar{A} \bar{T} \bar{T}$.

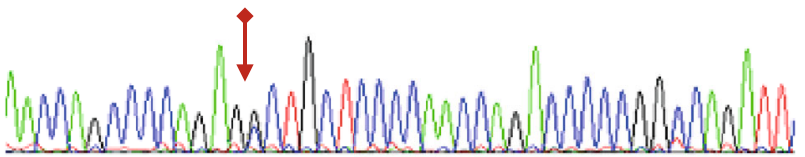

(b)
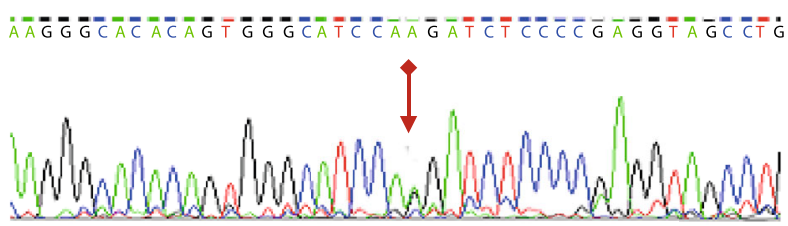

(c)

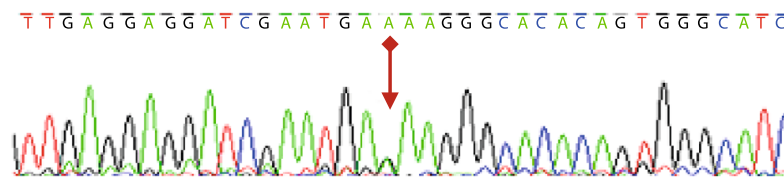

(d)

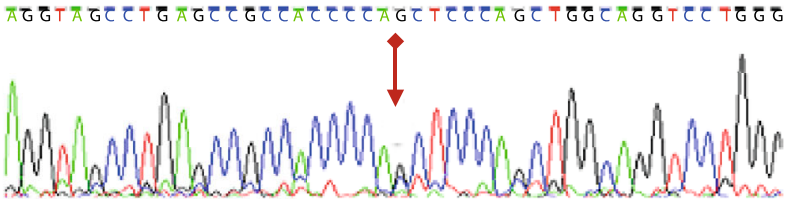

(e)

FIGURE 2: Five PRRT2 identified mutations in PKD/IC patients. (a) NM_1452392.2:c.650_670delinsCAATGGTGCCACCACTGGGTTA p.(Arg217Profs*8); (b) NM_1452392.2:c.412C>G p.(Pro138Ala); (c) NM_1452392.2:c.709G>A p.(Gly237Arg); (d) NM_ 1452392.2:c.686G>A p.(Arg229Lys); and (e) NM_1452392.2:c.743G>C p.(Ser 248Thr).

hotspot in this region. This mutation may be due to slippedstrand mechanism during DNA replication. Thus, it is a hotspot mutation $[8,32]$. The patient from family B (BII-1) presented a triple PRRT2 variants following genome sequencing of p.(Pro138Ala), p.(Arg229Lys), and p.(Gly237Arg) (Table 1). The double occurrence of PRRT2 mutations in a patient with PKD has been previously reported with intelligent disability [20]. While several studies reported that homozygous mutations cause sever neurological symptoms, recent reported c.981C $>\mathrm{G}$ homozygous mutation has not resulted in severe disorder [33]. Consistent with our result, this supports the hypothesis that differences in the epilepsy phenotypes in the patients with PRRT2 mutations depend on the types of PRRT2 mutations. We further screened parents of the patients for PRRT2 gene mutations and detected p.(Pro138Ala) and p.(Arg229Lys) variants in the unaffected mother of the patient (BI-2). This finding shows deficient mutation penetrance. However, the presence of p.(Gly237Arg) variant in both parents was not verified. The de novo result may endorse this mutation, which is consistent with genetic hereditary (Figure 1), in which putative mutation occurs de novo in the affected child of unaffected parents. Such a finding is in accordance with results of a previous study that presented the de novo mutagenesis of PRRT2 mutations in Chinese PKD, PKD/IC, and BFIS2 [32]. Moreover, p.(Pro138Ala) was reported in 15 Chinese sporadic PKD patients and in 14 patients affected with epileptic febrile seizures; its occurrence is seen as a loss of functional mutation [12]. PRRT2 p.(Pro138Ala) and p.(Gly237Arg) are predicted to be nonpathogenic and probably damaged by polyphen-2. Both are classified to be tolerated according to SIFT [2]. NM_1452392.2: c.412C>G is probably a causative mutation of $\mathrm{PKD} / \mathrm{IC}$. The proband in family C (CII-1) identified one previously unknown substitution variant NM_1452392.2: c.686G>A p.(Arg229 Lys) and previously reported NM_1452392.2: c.743G $>C$ p.(Ser248Thr) variant (Table 1). The NM_1452392.2: c.686G $>$ A p.(Arg229 Lys) is inherited from the father of the patient with cosegregation consistence. However, the NM_ 1452392.2: c.743G $>$ C p.(Ser248Thr) variant was reported by the 1000 genome project, ExAC, HapMap, and Exome sequencing project (accession number rs 747687177). The 
unaffected mother passed away and was unavailable for sequence analysis. However, the pathogenicity of NM_ 1452392.2:c.686G>A p.(Arg229 Lys) cannot be proven and it is predicted to be benign by polyphen-2. Recent study reported a patient with a benign missense variant in the PRRT2 gene (c.501C>T; p.Thr167Ile). The variant was detected in the normal phenotype proband's father, but the proband showed infrequent clinical features that have not been previously reported in patients with the same mutation [34]. Consistently, our result promotes suggestion that an epigenetic role involvement during fetal development may result in severe clinical expressions [34]. The pathogenicity is more prevalent in PKD patients, and c.612dupA was found to cause loss of interaction between PRRT2 and syntaxin IB (STXIB) [35]. The cosegregation of NM_1452392.2:c.650_ 670delins CAATGGTGCCACCACTGGGTTA and NM_ 1452392.2:c.686G > A mutations in family A and family $\mathrm{C}$ is consistent with the autosomal dominant mode of inheritance of PKD/IC [2]. These results underpin the conceivable part of NM_1452392.2: c.686G>A in the pathogenicity. The PRRT2 variants may be involved in the aetiology of PKD/IC disease. Recent studies of PKD highlight the role of PRRT2 variants and possibly contribute to the pathogenesis of PKD [35, 36]. Additionally, there is a need to clarify the relation of p.(Arg229 Lys) with the pathogenesis of PKD/IC and with other PRRT2-related phenotypes. Only 3 individuals, including 1 unaffected familial and 2 sporadic patients, were negative for PRRT2 mutations in addition to 50 geographically healthy controls. Clearly, the sporadic negative screening patients were most likely misdiagnosed with PKD/IC because of clinical indications, or the patients possess a mutation in another gene [21]. The patients may possess a disease that is different from or similar to typical PKD/IC $[6,15,32]$. The study mainly researched on genomic mutations in PRRT2 gene and more causal mutations and new findings may be discovered if genomic variation from whole-exome sequencing data or whole-genome sequencing data is used in the future studies.

\section{Conclusion}

The study identified the genetic variations in PRRT2 associated with $\mathrm{PKD} / \mathrm{IC}$ and uncovered new findings that indicate a novel mutation. Eleven patients of $\mathrm{PKD} / \mathrm{IC}$, i.e., 2 unaffected relatives and 50 healthy matched controls, were analyzed for PRRT2 mutations. Several previously identified variants and one novel variant were found.

In one family (A), the NM_1452392.2: c.650_670delins CAATGGTGCCACCACTGGGTTA PRRT2 mutation was identified as a causative mutation. Additionally, PRRT2 gene screen confirmed the presence of de novo mutation in the PKD patients, which may help clarify the mechanism of PKD. A novel sequence variant NM_1452392.2: c.686G $>$ A p.(Arg229 Lys) in two families and two sporadic cases of $\mathrm{PKD} / \mathrm{IC}$ are identified. These findings expand mutation spectrum of PRRT2. Detected PRRT2 mutations accounted for $88 \%$ and $50 \%$ in the investigated familial and sporadic cases of PKD/IC, respectively. Further studies involving functional experiments with more PKD pedigrees need to be conducted to reveal the pathogenicity of the detected variants and to clarify the molecular mechanisms by which these genetic variants alter human neural circuitry.

\section{Data Availability}

The data are shared with other partners, and available via contacting corresponding author, Jin-Lin Zhu (13845414150@139.com)

\section{Ethical Approval}

This study was approved by the local ethical committee of the Central Hospital of Jiamusi.

\section{Conflicts of Interest}

The authors declare that they have no competing interest.

\section{Authors' Contributions}

Salem Baldi and Jin-Ling Zhu contributed equally to this work.

\section{Acknowledgments}

The authors are grateful to all the teachers of the Biology and Anatomy departments of Jiamusi University and all the doctors of the Epilepsy and Paediatric departments of Central Hospital of Jiamusi for their generous support. This study was supported by Jiamusi University and innovate team project-number CXTDPY-201602, and Jiamusi University of Science and Technology project, number Sz2013-001.

\section{References}

[1] R. Caraballo, S. Pavek, A. Lemainque et al., "Linkage of benign familial infantile convulsions to chromosome 16p12-q12 suggests allelism to the infantile convulsions and choreoathetosis syndrome," The American Journal of Human Genetics, vol. 68, no. 3, pp. 788-794, 2001.

[2] W.-J. Chen, Y. Lin, Z.-Q. Xiong et al., "Exome sequencing identifies truncating mutations in _PRRT2_ that cause paroxysmal kinesigenic dyskinesia," Nature Genetics, vol. 43, no. 12, pp. 1252-1255, 2011.

[3] J.-L. Wang, L. Cao, X.-H. Li et al., "Identification of PRRT2 as the causative gene of paroxysmal kinesigenic dyskinesias,", Brain, vol. 134, no. 12, pp. 3493-3501, 2011.

[4] H.-Y. Lee, Y. Huang, N. Bruneau et al., "Mutations in the gene PRRT2 cause paroxysmal kinesigenic dyskinesia with infantile convulsions," Cell Reports, vol. 1, no. 1, pp. 2-12, 2012.

[5] C. Cai, O. Shi, and W.-D. Li, "Missense mutations of the proline-rich transmembrane protein 2 gene cosegregate with mild paroxysmal kinesigenic dyskinesia and infantile convulsions in a Chinese pedigree," Parkinsonism \& Related Disorders, vol. 19, no. 3, pp. 402-403, 2013.

[6] L. Cao, X.-J. Huang, L. Zheng, Q. Xiao, X.-J. Wang, and S.D. Chen, "Identification of a novel PRRT2 mutation in patients with paroxysmal kinesigenic dyskinesias and c. 649dupC as a mutation hot-spot," Parkinsonism \& Related Disorders, vol. 18, no. 5, pp. 704-706, 2012. 
[7] P. Hedera, J. Xiao, A. Puschmann, D. Momčilović, S. W. Wu, and M. S. LeDoux, "Novel PRRT2 mutation in an AfricanAmerican family with paroxysmal kinesigenic dyskinesia," BMC Neurology, vol. 12, no. 1, 2012.

[8] S. E. Heron, B. E. Grinton, S. Kivity et al., "PRRT2 mutations cause benign familial infantile epilepsy and infantile convulsions with choreoathetosis syndrome," The American Journal of Human Genetics, vol. 90, no. 1, pp. 152-160, 2012.

[9] A. Ishii, S. Yasumoto, Y. Ihara et al., "Genetic analysis of PRRT2 for benign infantile epilepsy, infantile convulsions with choreoathetosis syndrome, and benign convulsions with mild gastroenteritis," Brain and Development, vol. 35, no. 6, pp. 524-530, 2013.

[10] Q. Liu, Z. Qi, X.-H. Wan et al., "Mutations in PRRT2 result in paroxysmal dyskinesias with marked variability in clinical expression," Journal of Medical Genetics, vol. 49, no. 2, pp. 79-82, 2012.

[11] J. Schubert, R. Paravidino, F. Becker et al., "PRRT2 mutations are the major cause of benign familial infantile seizures," Human Mutation, vol. 33, no. 10, pp. 1439-1443, 2012.

[12] J. Youn, J. S. Kim, M. Lee et al., "Clinical manifestations in paroxysmal kinesigenic dyskinesia patients with proline-rich transmembrane protein 2 gene mutation," Journal of Clinical Neurology, vol. 10, no. 1, pp. 50-54, 2014.

[13] Z.-W. He, J. Qu, Y. Zhang et al., "PRRT2 mutations are related to febrile seizures in epileptic patients," International Journal of Molecular Sciences, vol. 15, no. 12, pp. 23408-23417, 2014.

[14] W. Zheng, J. Zhang, X. Deng et al., "Identification of a premature termination mutation in the proline-rich transmembrane protein 2 gene in a Chinese family with febrile seizures," Molecular Neurobiology, vol. 53, no. 2, pp. 835-841, 2016.

[15] I. E. Scheffer, B. E. Grinton, S. E. Heron et al., "PRRT2 phenotypic spectrum includes sporadic and fever-related infantile seizures," Neurology, vol. 79, no. 21, pp. 2104-2108, 2012.

[16] C. Castiglioni, I. López, F. Riant, E. Bertini, and A. Terracciano, "PRRT2 mutation causes paroxysmal kinesigenic dyskinesia and hemiplegic migraine in monozygotic twins," European Journal of Paediatric Neurology, vol. 17, no. 3, pp. 254-258, 2013.

[17] C. Marini, V. Conti, D. Mei et al., "PRRT2 mutations in familial infantile seizures, paroxysmal dyskinesia, and hemiplegic migraine," Neurology, vol. 79, no. 21, pp. 2109-2114, 2012.

[18] F. Riant, E. Roze, C. Barbance et al., "PRRT2 mutations cause hemiplegic migraine," Neurology, vol. 79, no. 21, pp. 21222124, 2012.

[19] U.-M. Sheerin, M. Stamelou, G. Charlesworth et al., "Migraine with aura as the predominant phenotype in a family with a PRRT2 mutation," Journal of Neurology, vol. 260, no. 2, pp. 656-660, 2013.

[20] L. Tan, K. Methawasin, E. Teng et al., "Clinico-genetic comparisons of paroxysmal kinesigenic dyskinesia patients with and without PRRT2 mutations," European Journal of Neurology, vol. 21, no. 4, pp. 674-678, 2014.

[21] D. Liu, Y. Zhang, Y. Wang et al., "Novel Locus for Paroxysmal Kinesigenic Dyskinesia Mapped to Chromosome 3q28-29," Scientific Reports, vol. 6, no. 1, 2016.

[22] H. Torisu, K. Watanabe, K. Shimojima et al., "Girl with a PRRT2 mutation and infantile focal epilepsy with bilateral spikes," Brain and Development, vol. 36, no. 4, pp. 342-345, 2014.
[23] A. Gkampeta, L. Fidani, J. Clarimón et al., "Association of brain-derived neurotrophic factor (BDNF) and elongator protein complex 4 (ELP4) polymorphisms with benign epilepsy with centrotemporal spikes in a Greek population," Epilepsy Research, vol. 108, no. 10, pp. 1734-1739, 2014.

[24] F. Reimann, J. J. Cox, I. Belfer et al., "Pain perception is altered by a nucleotide polymorphism in SCN9A," Proceedings of the National Academy of Sciences, vol. 107, no. 11, pp. 51485153, 2010.

[25] X.-R. Liu, M. Wu, N. He et al., "NovelPRRT2mutations in paroxysmal dyskinesia patients with variant inheritance and phenotypes," Genes Brain and Behavior, vol. 12, no. 2, pp. 234240, 2013.

[26] W.-L. Lee, A. Tay, H.-T. Ong, L.-M. Goh, A. P. Monaco, and P. Szepetowski, "Association of infantile convulsions with paroxysmal dyskinesias (ICCA syndrome): confirmation of linkage to human chromosome 16p12-q12 in a Chinese family," Human Genetics, vol. 103, no. 5, pp. 608-612, 1998.

[27] P. Szepetowski, J. Rochette, P. Berquin, C. Piussan, G. M. Lathrop, and A. P. Monaco, "Familial infantile convulsions and paroxysmal choreoathetosis: a new neurological syndrome linked to the pericentromeric region of human chromosome 16," The American Journal of Human Genetics, vol. 61, no. 4, pp. 889-898, 1997.

[28] Y.-T. Liu, F.-S. Nian, W.-J. Chou et al., "PRRT2 Mutations Lead to Neuronal Dysfunction and Neurodevelopmental Defects," Oncotarget, vol. 7, no. 26, pp. 39184-39196, 2016.

[29] P. Valente, E. Castroflorio, P. Rossi et al., "PRRT2 Is a Key Component of the Ca2+-Dependent Neurotransmitter Release Machinery," Cell Reports, vol. 15, no. 1, pp. 117-131, 2016.

[30] Y.-C. Lee, M.-J. Lee, H.-Y. Yu et al., "PRRT2 mutations in paroxysmal kinesigenic dyskinesia with infantile convulsions in a Taiwanese cohort," PLoS One, vol. 7, no. 8, article e38543, 2012.

[31] A. J. A. Groffen, T. Klapwijk, A.-F. van Rootselaar, J. L. Groen, and M. A. J. Tijssen, "Genetic and phenotypic heterogeneity in sporadic and familial forms of paroxysmal dyskinesia," Journal of Neurology, vol. 260, no. 1, pp. 93-99, 2013.

[32] H. F. Li, W. Ni, Z. Q. Xiong, J. Xu, and Z. Y. Wu, "PRRT2 c. 649dupC mutation derived from de novo in paroxysmal kinesigenic dyskinesia," CNS Neuroscience \& Therapeutics, vol. 19, no. 1, pp. 61-65, 2013.

[33] A. Okumura, K. Shimojima, H. Kurahashi et al., "PRRT2 mutations in Japanese patients with benign infantile epilepsy and paroxysmal kinesigenic dyskinesia," Seizure, vol. 71, pp. 1-5, 2019.

[34] P. Pavone, G. Corsello, S. Y. Cho et al., "PRRT2 gene variant in a child with dysmorphic features, congenital microcephaly, and severe epileptic seizures: genotype-phenotype correlation?," Italian Journal of Pediatrics, vol. 45, no. 1, pp. 159159, 2019.

[35] H. Ma, S. Feng, X. Deng et al., “APRRT2variant in a Chinese family with paroxysmal kinesigenic dyskinesia and benign familial infantile seizures results in loss of interaction withSTX1B," Epilepsia, vol. 59, no. 8, pp. 1621-1630, 2018.

[36] J. G. Lu, J. Bishop, S. Cheyette et al., “A novelPRRT2pathogenic variant in a family with paroxysmal kinesigenic dyskinesia and benign familial infantile seizures," Molecular Case Studies, vol. 4, no. 1, p. a002287, 2018. 